\title{
DESIGN OF A PULSED X-RAY SYSTEM FOR FLUORESCENT LIFETIME MEASUREMENTS WITH A TIMING ACCURACY OF 109 PS*
}

\author{
S. E. Derenzo, W. W. Moses, and S. C. Blankespoor \\ Lawrence Berkeley Laboratory, University of California, Berkeley, CA 94720 \\ M. Ito, and K. Oba \\ Hamamatsu Photonics K.K., Hamamatsu City, Japan
}

LBL --33354

\begin{abstract}
We describe the design of a table-top pulsed $x$-ray system for measuring fluorescent lifetime and wavelength spectra of samples in both crystal and powdered form. The novel element of the system is a light-excited $x$-ray tube with a tungsten anode at $+30 \mathrm{kV}$ potential. The $S-20$ photocathode is excited by a laser diode with a maximum rate of $10 \mathrm{MHz}$, each pulse having $<100 \mathrm{ps}$ fwhm (full-width at halfmaximum) and $>10^{7}$ photons. In a collimated $2 \mathrm{~mm} \times 2 \mathrm{~mm}$ beam spot $40 \mathrm{~mm}$ from the anode we expect $>1 \mathrm{x}$-ray per pulse. A sample is exposed to these $x$-rays and fluorescent photons are detected by a microchannel PMT with a photoelectron transit time spread of $60 \mathrm{ps}$ fwhm, a sapphire window, and a bialkali photocathode (wavelength range 180-600 $\mathrm{nm})$. The combined time spread of a laser diode, the $\mathrm{x}$-ray tube, and a microchannel tube has been measured to be 109 ps fwhm. To measure wavelength spectra, a reflection grating monochromator is placed between the sample and the PMT.
\end{abstract}

\section{BACKGROUND AND INTRODUCTION}

In previous work, an electron synchrotron was used in single-bunch mode to measure the $\mathrm{x}$-ray excited fluorescence of over 400 compounds. Interesting fluorescent emissions were detected from several compounds by this method, including $\mathrm{CeF}_{3}, \mathrm{PbCO}_{3}, \mathrm{PbSO}_{4}, \mathrm{Yb}_{2} \mathrm{O}_{3}, \mathrm{CeCl}_{3}, \mathrm{BaCl}_{2}$, and $\mathrm{CuI}$ $[1,2]$. At the same time, a light-excited $x$-ray tube was developed for producing very brief $(\approx 100 \mathrm{ps})$ pulses of $x$-rays (Figure 1) [3].

In this work, we describe the design of a table-top pulsed $x$-ray fluorescence measurement system using a laser diode and a light-excited $x$-ray tube. This system has a number of advantages over the electron synchrotron technique. Among these are (i) measuring samples without having to transport personnel and equipment to a remote site, (ii) measuring samples immediately rather than waiting for the next single-bunch beam run, (iii) a narrower $x$-ray pulse width $(<100 \mathrm{ps})$, and (iv) the ability to vary the excitation repetition period from $100 \mathrm{~ns}$ to well beyond $10 \mu \mathrm{s}$.

* This work was supported in nnrt by the U.S. Department of Energy contract DE-AC03-76SF( 098, in part by Public Health Service Grants Nos. P01 25840, R01 CA48002, and GM0815507, and in part by The Whitaker Foundation.

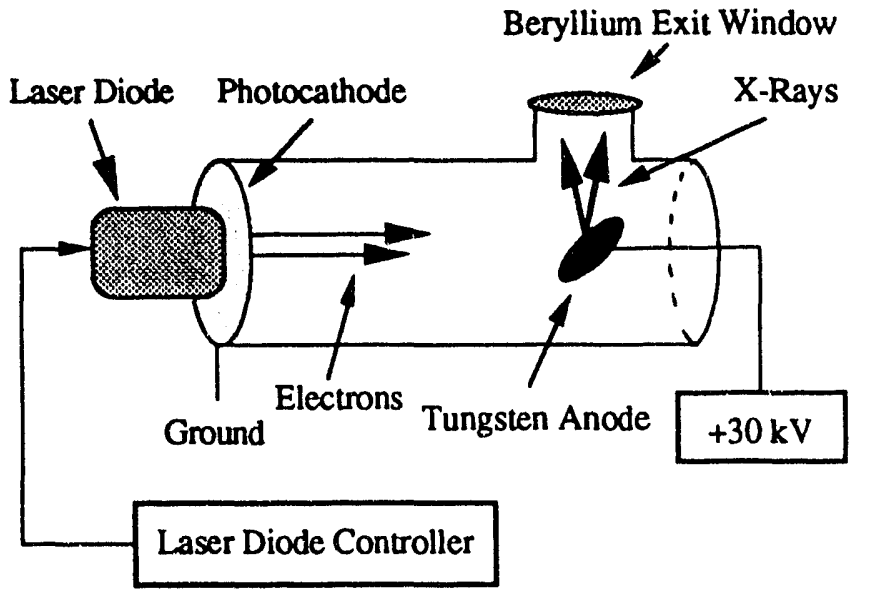

Figure 1. Schematic diagram of the pulsed $x$-ray source. $A$ laser diode excites the photocathode with 60 ps pulses at $\leq 10 \mathrm{MHz}$ and the photoelectrons are accelerated toward the tungsten anode at 30 $\mathrm{kV}$ potential to produce $x$-rays.

The method of Bollinger and Thomas [4] (as modified by Moszynski and Bengtson [5] for coincident $511 \mathrm{keV}$ annihilation photons) is also used to measure fluorescent decay spectra. However, the timing accuracy is limited to about $200 \mathrm{ps}$ fwhm by jitter in the trigger scintillator, and it is difficult to determine the fraction of slow components.

\section{PULSED X-RAY SOURCE}

\subsection{Laser Diode}

The PLP-01 Picosecond Light Pulser consists of a controller and a laser diode head. The pulse width and number of photons per pulse depend only weakly on the pulse rate, which can be varied from $1 \mathrm{~Hz}$ to $10 \mathrm{MHz}$. Each light pulse is preceded by a timing pulse with a time jitter of \pm 10 ps. In addition, the laser diode can be extemally triggered or operated in dc mode. See Table 1 for additional details.

Table 1. Characteristics of Hamamatsu PLP-01 Light Pulser with C3551-01 Controller and LDH065 Laser Diode Head

\begin{tabular}{ll}
\hline Emission wavelength & $650 \mathrm{~nm}$ \\
Peak power & $>100 \mathrm{~mW}$ \\
Pulse width & $50-100 \mathrm{ps} \mathrm{fwhm}$ \\
Pulse repetition rate & $\mathrm{dct} 10 \mathrm{MHz}$ \\
Photons per pulse & $>10^{7}$ \\
Timing pulse jitter & $\pm 10 \mathrm{ps}$ \\
\hline & \\
&
\end{tabular}




\subsection{Light-excited X-ray Tube}

The light-excited $x$-ray tube (Figure 1), is essentially a single-stage photomultiplier tube. It has an S-20 photocathode at ground potential and a tungsten anode at $+30 \mathrm{kV}$. Light liberates photoelectrons at the photocathode, which are accelerated to $30 \mathrm{keV}$ to strike the tungsten target, producing bremsstrahlung $\mathrm{x}$-rays. The photocathode to anode transit time is $0.8 \mathrm{~ns}$ and the anode surface is oriented at $45^{\circ}$ with respect to the incident electron beam. See Table 2 for additional details.

The maximum anode current of $50 \mu \mathrm{A}$ corresponds to a maximum photon rate of $3 \times 10^{15} / \mathrm{s}$ at $10 \%$ quantum efficiency. At $10 \mathrm{MHz}$ and $2 \times 10^{7}$ photons per pulse, the laser diode will provide $2 \times 10^{14}$ photons $/$.

Table 2. Characteristics of Hamamatsu N5084 Light-excited X-Ray Tube

\begin{tabular}{ll}
\hline Overall tube length & $152 \mathrm{~mm}$ \\
Tube Diameter & $52 \mathrm{~mm}$ \\
Photocathode & $\mathrm{S}-20$ \\
Quantum efficiency @ 650 nm & $>10 \%$ \\
Photocathode diameter & $12 \mathrm{~mm}$ \\
Target material (anode) & Tungsten $\left(45^{\circ}\right)$ \\
Output window material & Beryllium \\
Output window diameter & $20 \mathrm{~mm}$ \\
Output window thickness & $0.5 \mathrm{~mm}$ \\
Cooling & Natural air \\
Tube voltage (max) & $30 \mathrm{kV}$ \\
Tube current (max) & $50 \mu \mathrm{A}$ \\
Photocathode photon rate (max) & $3 \times 10^{15} / \mathrm{s} @ 10 \%$ QE \\
\hline
\end{tabular}

\section{RESULTS}

\subsection{Measurement of Timing Response}

The light-excited $x$-ray tube was tested by illuminating its photocathode with laser diode pulses and measuring the $x$-rays with a Be-window microchannel electron multiplier (Figure 2). The laser diode was coupled to the $\mathrm{x}$-ray tube with an optical fiber. The measured time distribution of detected $x$-rays is shown in Figure 3. The 109 ps fwhm includes three components: the width of the laser diode pulses $(55 \mathrm{ps})$, time spread in the light-excited $x$-ray tube, and the timing resolution of the microchannel tube ( $57 \mathrm{ps}$ ). The contribution of the lightexcited $\mathrm{x}$-ray tube is inferred to be $75 \mathrm{ps}$.

\subsection{System for Fluorescence Measurements}

Figure 4 shows the system for measuring fluorescence decay timing spectra from powder or crystal samples. The delayed coincidence method is used to record the time distribution between the $\mathrm{x}$-ray pulses and the individual fluorescent photons.

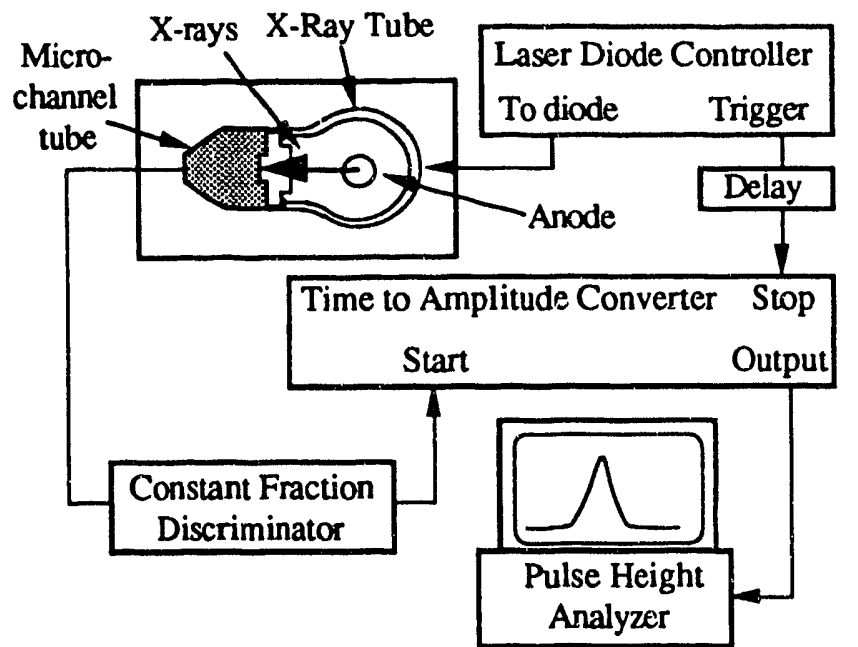

Figure 2. Method for measuring the combined time spread of laser diode, light-excited $x$-ray tube, and microchannel $x$-ray detector. The $\mathrm{x}$-rays pass through $\mathrm{Be}$ windows to interact directly with the microchannel multiplier.

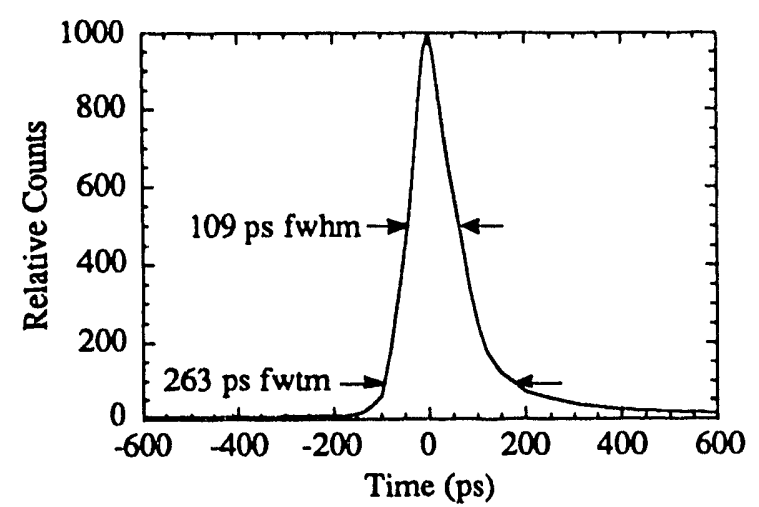

Figure 3. Combined time spread of $109 \mathrm{ps}$ (fwhm) due to width of laser diode pulse $(55 \mathrm{ps})$, and time spreads in the light-excited $\mathrm{x}$ ray tube $(75 \mathrm{ps})$ and microchannel $x$-ray detector $(57 \mathrm{ps})$. The spectrum was measured with the setup shown in Figure 2, using an $x$-ray tube voltage of $10 \mathrm{kV}$.

The laser diode head is proximity coupled to the $\mathrm{x}$-ray tube photocathode for maximum photon rate. The microchannel PMT has a bialkali photocathode, a sapphire window, a wavelength range from 180 to $600 \mathrm{~nm}$, and a $60 \mathrm{ps}$ transit time spread. The PMT is thermoelectrically cooled to $-20^{\circ} \mathrm{C}$ to reduce the dark rate to a few photoelectrons/s.

Emission spectra can be measured by inserting a monochromator between the sample and the microchannel phototube. To extend the upper limit to $900 \mathrm{~nm}$, a phototube with a quartz window, a GaAs photocathode, and 4 ns transit time spread can be used.

The sample chamber is evacuated to a pressure below $10^{-3}$ $\mathrm{mm} \mathrm{Hg}$ to avoid fluorescence from air. A current meter at the photocathode is used to monitor the photoelectron current and a current meter at the power supply is used to monitor the sum of the anode current and any leakage currents to ground. 


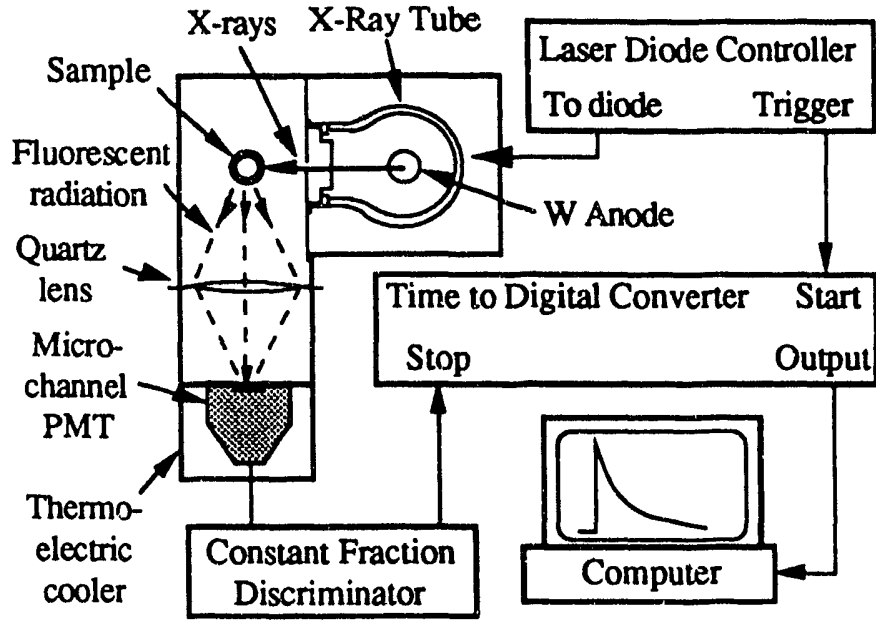

Figure 4. System for exciting samples with $<100$ ps pulses of $x$ rays and measuring the fluorescent photons with a microchannel phototube. See text for details.

For measuring the system time response for the fluorescence time measurement system shown in Figure 4, we plan to position the microchannel PMT in the $x$-ray beam at an angle to produce photoelectrons at the photocathode. This measurement includes (i) the laser diode pulse shape, (ii) time spread in the $x$-ray tube, (iii) photoelectron time spread in the PMT between the photocathode and the microchannel plate and (iv) electron time spread in the microchannel multiplier.

Safety interlocks allow the high voltage power supply to be turned on only after the high voltage cables and sample chamber are connected and the sample chamber is evacuated. The high voltage supply trips off whenever the current exceeds $50 \mu \mathrm{A}$ or an ionization chamber monitor exceeds a set limit.

The system shown in Figure 4 has a parts cost of about $\$ 50,000$ (U.S.), with the light-excited $x$-ray tube, the microchannel PMT, and the laser diode being the most expensive components.

\subsection{Data Acquisition and Analysis}

Time differences between the $\mathrm{x}$-ray excitation and the arrival of fluorescent photons are digitized by a time to digital converter (TDC) with a bin width of $50 \mathrm{ps}$ and a maximum dynamic range of $800 \mu \mathrm{s}$, corresponding to the maximum 24bit CAMAC data number. The TDC can detect multiple photon detections in the conversion time range, and these events are rejected to reduce bias and permit substantially higher data rates [6]. A compression algorithm preserves the $50 \mathrm{ps}$ bin width near the time of the excitation but combines data into progressively wider bins at later times. In a typical case, $2 \times 10^{5}$ bins spanning a $10 \mu$ s conversion range are compressed into $2 \times 10^{3}$ bins for fitting. The fitting program determines fractions, decay times, and rise times of fluorescent components by fitting a sum of exponentials (convolved with the system timing response) to the compressed data [7].

\section{CONCLUSIONS}

By combining a state-of-the-art laser diode and a recently developed light-excited $x$-ray tube, we have designed a tabletop system for measuring fluorescent intensities and decay times with a timing accuracy of about 100 ps. The data acquisition uses photon counting and signal averaging, so that accurate measurements are possible for even weakly fluorescing samples. The system is more accurate than methods using coincident nuclear radiation and more accurate and convenient than methods using synchrotron $x$-radiation.

\section{ACKNOWLEDGEMENTS}

We would like to thank J. Cahoon and T. Vuletich for technical assistance, R. Muller for technical coordination, and P. Rentzepis and K. Kaufmann for helpful discussions.

This work was supported in part by the Director, Office of Energy Research, Office of Health and Environmental Research, Medical Applications and Biophysical Research Division of the U.S. Department of Energy under contract No. DE-AC03-76SF00098, and in part by Public Health Service Grant Nos. P01 HL25840, R01 CA48002, and GM08155-07, awarded by the National Heart Lung and Blood, National Cancer, and General Medical Institutes, Department of Health and Human Services. Two of us (S.C. Blankespoor and W.W. Moses) were supported in this, search by the Whitaker Foundation.

Reference to a company or product name does not imply approval or recommendation by the University of California or the U.S. Department of Energy to the exclusion of others that may be suitable.

\section{REFERENCES}

[1] S. E. Derenzo, W. W. Moses, R. Perera, et al., "Prospects for new inorganic scintillators," IEEE Trans Nucl Sci, vol. NS-37, pp. 203-208, 1990.

[2] S. E. Derenzo, W. W. Moses, J. L. Cahoon, et al., "X-ray fluorescence measurements of 412 inorganic compounds," IEEE Nuclear Science Symposium Conference Record 91CH3100-5, vol. 1, pp. 143-147, 1992.

[3] K. Oba, "Instrument to measure fluorescence which has occurred in a sample stimulated by X-rays," U.S. patent $\# 4,724,536$, issued to Hamamatsu Photonics, Japan, Feb 9. 1988.

[4] L. M. Bollinger and G. E. Thomas, "Measurement of the time dependence of scintillation intensity by a delayed. coincidence method," Rev Sci Instr, vol. 32, pp. 1044 . $1050,1961$.

[5] M. Moszynski and B. Bengtson, "Light pulse shaves from plastic scintillators," Nucl Instr Meth, vol. 142, pp. 417 434, 1977.

[6] W. W. Moses, "A method to increase scintillation lifetime measurement rates using a multi-hit TDC," Nucl Instr Meth (in preparation), 1993.

[7] S. Derenzo, "TAUFIT- a fitting program to determine fluorescent decay components as sum of exponentials convolved with an impulse response," Lawrence Berkeley Laboratory Report No. LBL-33050, 1993. 

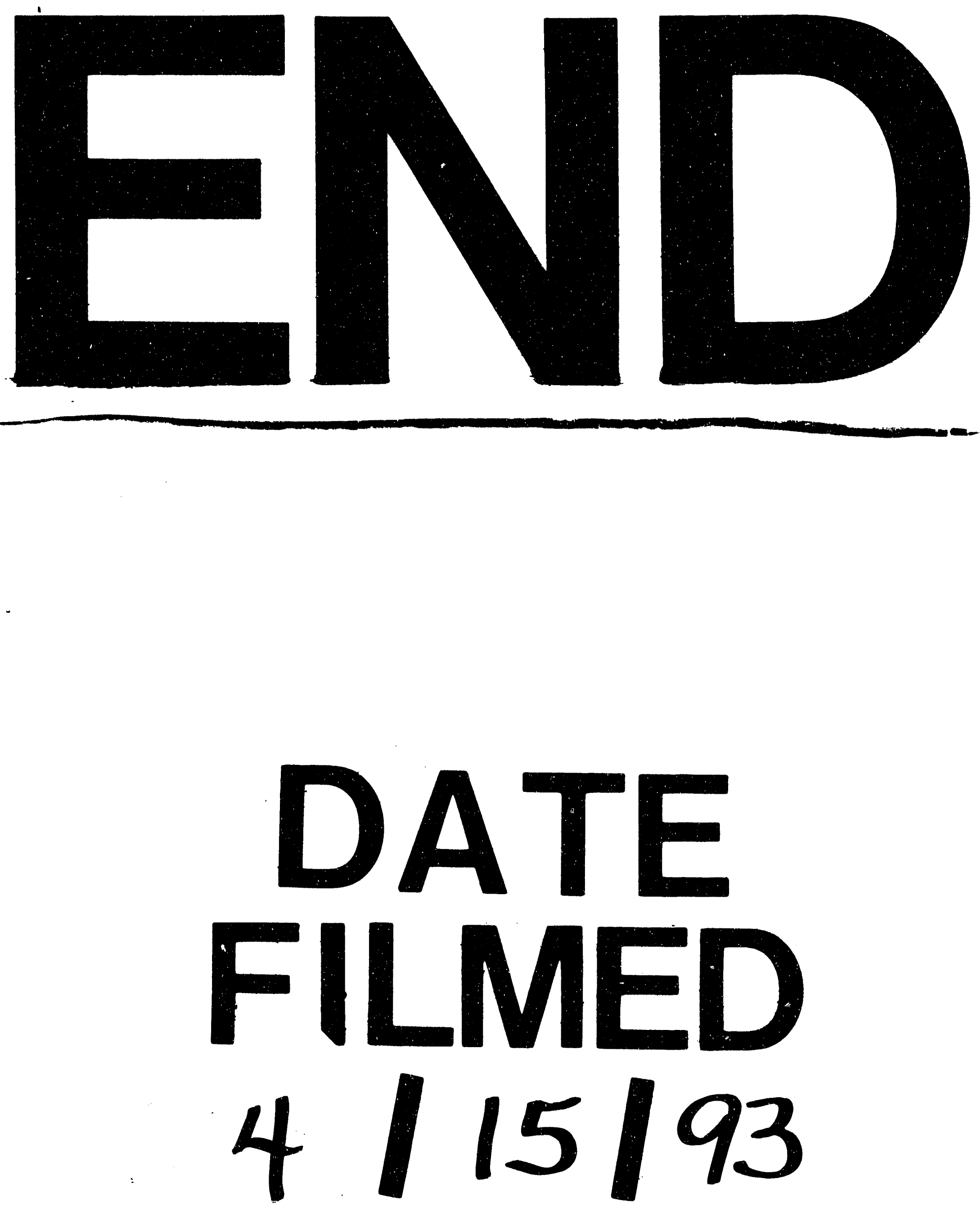
